\title{
Stress Measurements of Coarse Grain Materials using Double Exposure Method with Hard Synchrotron X-Rays
}

\author{
Kenji Suzuki ${ }^{1, a,{ }^{*}}$, Takahisa Shobu ${ }^{2, b}$ and Ayumi Shiro ${ }^{3, c}$ \\ ${ }^{1}$ Faculty of Education, Niigata University, Ikarashi-2-no-cho, Nishi-ku, Niigata, 950-2181, Japan \\ 2Japan Atomic Energy Agency, 1-1-1, Koto, Sayo-cho, Hyogo, 679-5148, Japan \\ ${ }^{3}$ National Institutes for Quantum and Radiological Science and Technology, 1-1-1, Koto, Sayo- \\ cho, Hyogo, 679-5148, Japan \\ asuzuki@ed.niigata-u.ac.jp, bshobu@sp8sun.spring8.or.jp, cshiro.ayumi@qst.go.jp
}

Keywords: X-Ray Stress Measurement, Coarse Grain, Double Exposure Method, Hard Synchrotron X-Ray

\begin{abstract}
The double exposure method (DEM) is proposed herein for X-ray stress measurement of coarse grain materials. A diffraction angle was obtained from an incident beam and a spotty diffracted beam. Each X-ray beam was measured by an area detector on a linear motion stage on a $2 \theta$-arm. To verify the effectiveness of the DEM, the residual stress of a plastically bent specimen and the residual stress distribution of an indented specimen was measured. The results obtained with the DEM were similar to the results of simulations using the finite element method confirming that the DEM is useful for X-ray stress measurements of coarse grain materials.
\end{abstract}

\section{Introduction}

There are three X-ray stress measurement methods that use area detectors: the 2D method [1], the $\cos \alpha$ method [2], and the direct-method [3]. However, these methods cannot be applied to the stress measurement of coarse grain materials. Therefore, a diffraction spot trace method (DSTM) has been developed and employed for strain scanning coarse grain materials [4]. However, as a rotating-slit system is indispensable, the DSTM involves a complicated experimental system. The details of the rotating-slit system were described in a previous study [5].

Herein, we propose a double exposure method (DEM), which does not require a slits system and is therefore suitable for the stress measurement of coarse grain materials.

\section{Double Exposure Method}

The difficulties in the stress measurement of coarse grain materials are as follows:

1. The diffraction pattern from a coarse grain is spotty.

2. The diffraction angle cannot be determined from the diffraction centre because the diffraction centre depends on each grain.

3. The measured diffraction angle of the coarse grain is affected by the divergence of the incident beam.

These problems are solved by using an area detector, a DEM, and synchrotron radiation. Usually, a diffraction angle is measured by a diffractometer or by using a tangent, but these methods cannot be applied to coarse grains. In the DEM proposed herein, the diffraction angle is determined from two lines consisting of the incident X-ray beam and the diffracted beam.

Figure 1 shows the configuration and coordinate system of the DEM. As shown in Fig. 1 (a), the specimen is set on a sample stage, and the area detector (PILATUS) is mounted on a linear motion stage on a $2 \theta$-arm. The diffraction spots from transmitted X-rays are measured by an area 


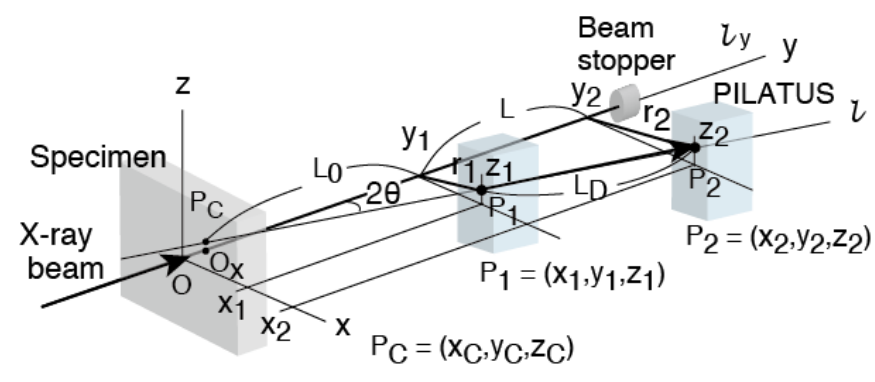

(a) DEM

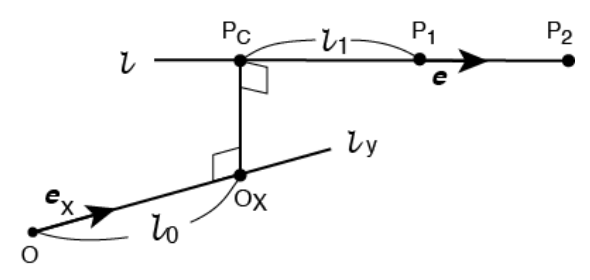

(b) Intersection of straight lines

Fig. 1 Configuration and geometry of double exposure method (DEM).

detector. The straight line of the incident beam, $l_{\mathrm{y}}$, is determined by the area detector and is defined as the $y$-axis.

When a diffraction spot is measured with the area detector, the straight line of the diffraction spot is given by the two points $\mathrm{P}_{1}\left(x_{1}, y_{1}, z_{1}\right)$ and $\mathrm{P}_{2}\left(x_{2}, y_{2}, z_{2}\right)$. The radii $r_{1}$ and $r_{2}$ are given by

$$
r_{1}=\sqrt{x_{1}^{2}+y_{1}^{2}}, \quad r_{2}=\sqrt{x_{2}^{2}+y_{2}^{2}} .
$$

The diffraction angle $2 \theta$ is obtained from the following relation.

$$
2 \theta=\arctan \left(\frac{r_{2}-r_{1}}{y_{2}-y_{1}}\right) .
$$

The straight line $l$ is the diffracted beam and passes through $\mathrm{P}_{1}$ and $\mathrm{P}_{2}$. The unit vectors of the straight lines $l_{\mathrm{y}}$ and $l$ in Fig. 1 (b) are given by

$$
\boldsymbol{e}_{X}=(0,1,0), \quad \boldsymbol{e}=\frac{\left(x_{2}-x_{1}, y_{2}-y_{1}, z_{2}-z_{1}\right)}{\left\|L_{D}\right\|} .
$$

As shown in Fig. 1 (b), the line segment between the points $\mathrm{P}_{\mathrm{C}}$ and $\mathrm{O}_{\mathrm{X}}$ is perpendicular to both straight lines; therefore, the inner products become zero.

$$
\boldsymbol{e} \cdot\left(\boldsymbol{O P _ { C }}-\boldsymbol{O O} \boldsymbol{O}_{X}\right)=\boldsymbol{e}_{X} \cdot\left(\boldsymbol{O P _ { C }}-\boldsymbol{O O} \boldsymbol{O}_{X}\right)=0 .
$$

The position vectors are given by

$$
\boldsymbol{O P _ { C }}=\boldsymbol{O} \boldsymbol{P}_{1}-l_{1} \boldsymbol{e}, \quad \boldsymbol{O} \boldsymbol{O}_{X}=l_{0} \boldsymbol{e}_{X} .
$$

By substituting Eq. 5 into Eq. 4, the lengths $l_{0}$ and $l_{1}$ are obtained as follows:

$$
\begin{aligned}
& l_{0}=y_{1}-\frac{\cot ^{2} 2 \theta}{L}\left[\left(x_{2}-x_{1}\right) x_{1}+\left(z_{2}-z_{1}\right) z_{1}\right] \\
& l_{1}=\frac{\cos 2 \theta}{L \sin ^{2} 2 \theta}\left[\left(x_{2}-x_{1}\right) x_{1}+\left(z_{2}-z_{1}\right) z_{1}\right]
\end{aligned}
$$

Finally, the position of the diffracted grain, $\mathrm{P}_{\mathrm{C}}\left(x_{C}, y_{C}, \mathrm{Z}_{C}\right)$, can be obtained from Eq. 5 and 7.

$$
\begin{aligned}
& x_{C}=x_{1}-\frac{\cot ^{2} 2 \theta}{L^{2}}\left[\left(x_{2}-x_{1}\right) x_{1}+\left(z_{2}-z_{1}\right) z_{1}\right]\left(x_{2}-x_{1}\right) \\
& y_{C}=y_{1}-\frac{\cot ^{2} 2 \theta}{L}\left[\left(x_{2}-x_{1}\right) x_{1}+\left(z_{2}-z_{1}\right) z_{1}\right]
\end{aligned}
$$




$$
z_{C}=z_{1}-\frac{\cot ^{2} 2 \theta}{L^{2}}\left[\left(x_{2}-x_{1}\right) x_{1}+\left(z_{2}-z_{1}\right) z_{1}\right]\left(z_{2}-z_{1}\right)
$$

\section{Synchrotron X-Ray Measurements and Test Specimens}

The stress measurements using the DEM were carried out at SPring-8 on the beamline BL22XU. A linear motion stage with a stroke length of $800 \mathrm{~mm}$ was set on the $2 \theta$-arm, and a PILATUS$300 \mathrm{~K}$ area detector was mounted on the linear motion stage. The $2 \theta$-arm was moved to $0^{\circ}$, and the beam centre was measured by the detector at two positions $\left(0, y_{1}, 0\right)$ and $\left(0, y_{2}, 0\right)$. The $2 \theta$-arm was moved to $26^{\circ}$, and the diffraction spots were measured at $\mathrm{P}_{1}$ and $\mathrm{P}_{2}$. In this experiment, $L_{D}$ was $791.889 \mathrm{~mm}, L_{0}$ was $707.989 \mathrm{~mm}$, and $L$ was $711.746 \mathrm{~mm}$.

The energy of the X-rays was $30.034 \mathrm{keV}$, and the dimensions of the incident beam was $0.2 \times$ $0.2 \mathrm{~mm}^{2}$. The effective area of the detector was $83.8 \times 106.5 \mathrm{~mm}^{2}$, the total number of pixels was $487 \times 619$ pixel, and the spatial resolution was $0.172 \times 0.172 \mathrm{~mm}^{2}$.

The test specimen was made of the aluminum alloy A5052 and was annealed at 623K for $1 \mathrm{~h}$. The mean grain size of the specimen was $27 \mu \mathrm{m}$. The Al 331 diffraction was employed, and the stress-free diffraction angle was $2 \theta_{0}=25.627^{\circ}\left(d_{0}=93.006 \mathrm{pm}\right)$. The stiffness of the single crystal $c_{i j}$ was obtained from another study [6]. The diffraction elastic constants were calculated with the Kröner model $[7,8]$ and are $E_{\mathrm{X}}=71.39 \mathrm{GPa}$ and $v_{\mathrm{X}}=0.347$ for the $\mathrm{Al} 331$ diffraction.

\section{Residual Stress of the Plastically Bent Specimen}

Diffraction images were measured with the PILATUS at $\mathrm{P}_{1}$ and $\mathrm{P}_{2}$, and examples are shown in Fig. 2 (a). The rectangular box in the $\mathrm{P}_{1}$ image indicates the detected area at $\mathrm{P}_{2}$. The diffraction spots from the 331 diffraction were used for the stress measurement. Each diffraction image was converted into a binary image, from which the outline of the diffraction spot was extracted.

As shown in Fig. 2 (b), the brightness centre of gravity was calculated, and the coordinates $\mathrm{P}_{1}\left(x_{1}, \mathrm{Z}_{1}\right)$ and $\mathrm{P}_{2}\left(x_{2}, \mathrm{Z}_{2}\right)$ of the diffraction spots were determined by the matching program. Finally, the diffraction angle $2 \theta$ and the diffraction position $\mathrm{P}_{C}\left(x_{C}, y_{C}, z_{C}\right)$ were obtained. As the process was very complicated, we developed an expert system that performed the analyses and matching mentioned above.
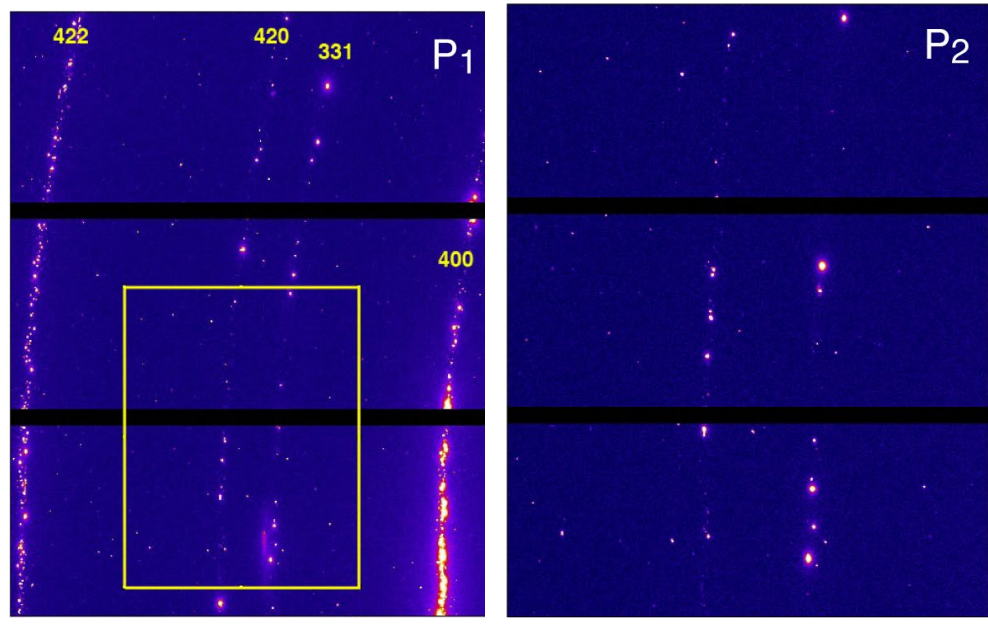

(a) Diffraction images
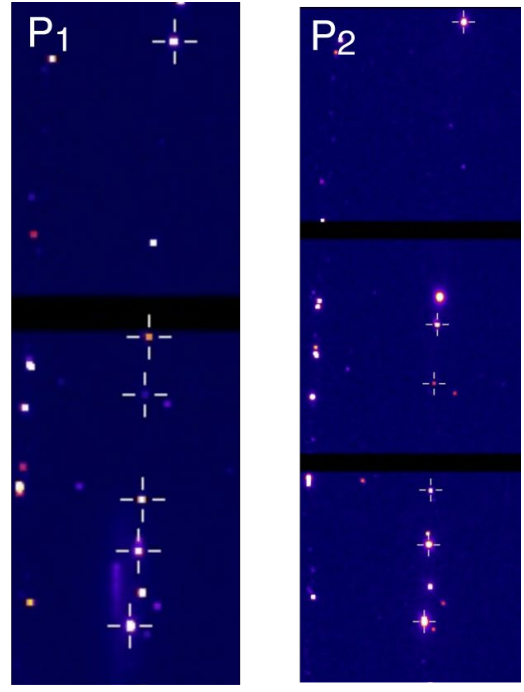

(b) Results of matching

Fig. 2 (a) Diffraction images measured by DEM and (b) matching diffraction spots at $P_{1}$ and $P_{2}$. 
The plate specimen was 4.7-mm wide and 3-mm thick and was plastically bent. The diffraction angle and position of the diffracted grain were measured using the DEM. Figure 3 (a) shows the measured diffraction angles. The direction along the width of the specimen is for $\chi=0$ and that of the longitudinal bar axis is for $\chi=90$. The error bars in the figure indicate the standard deviation. The diffraction angle along the longitudinal bar axis $(\chi=90)$ changes due to plastic bending. The plots in Fig. 3 (b) show the positions of the diffracted grains. The rectangle with the black dashed line in Fig. 3 (b) indicates the cross section of the specimen. The vertical red dashed lines correspond to the incident X-ray beam. The position of each coarse grain can be obtained by the DEM as shown in Fig. 3 (b). In the plastic zone, a diffraction ring appears, and the spotty pattern is less pronounced. Therefore, the number of points in the plastic zone decreases compared with the elastic zone.

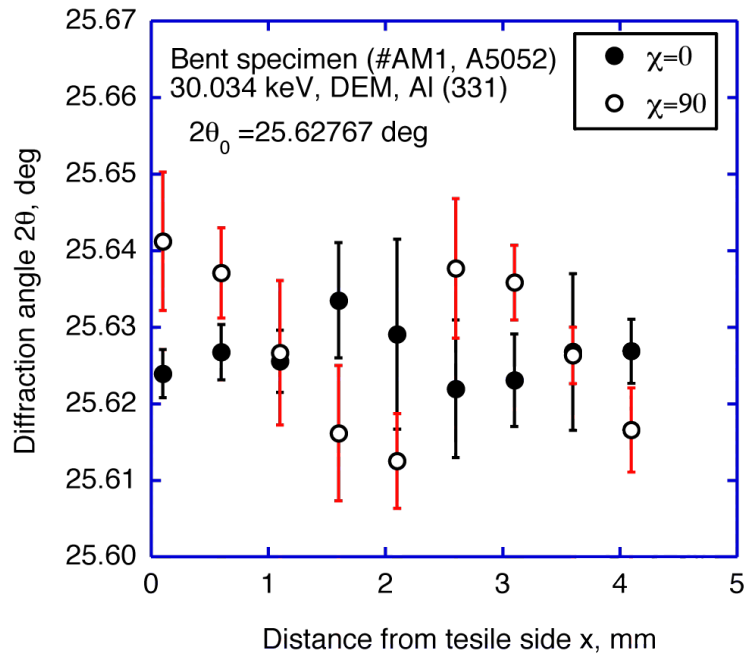

(a) Diffraction angle

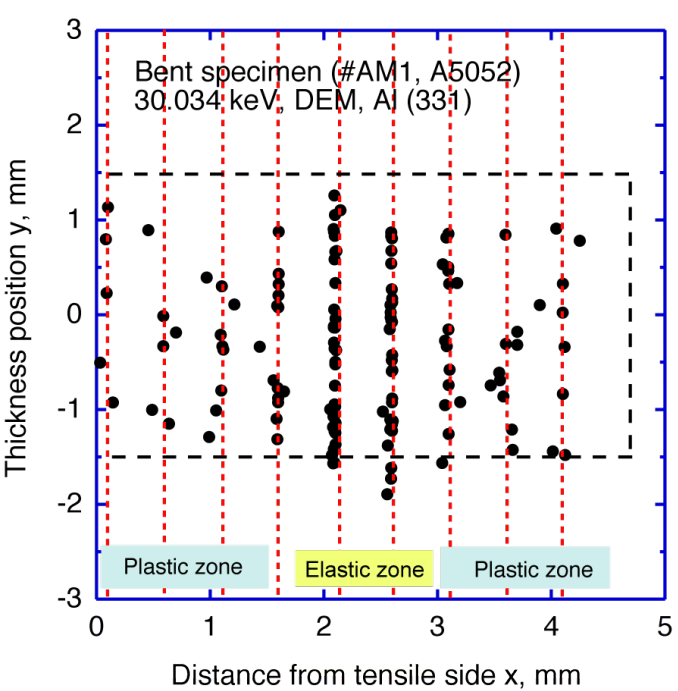

(b) Position of the diffracted grain

Fig. 3 Results of the plastically bent specimen.

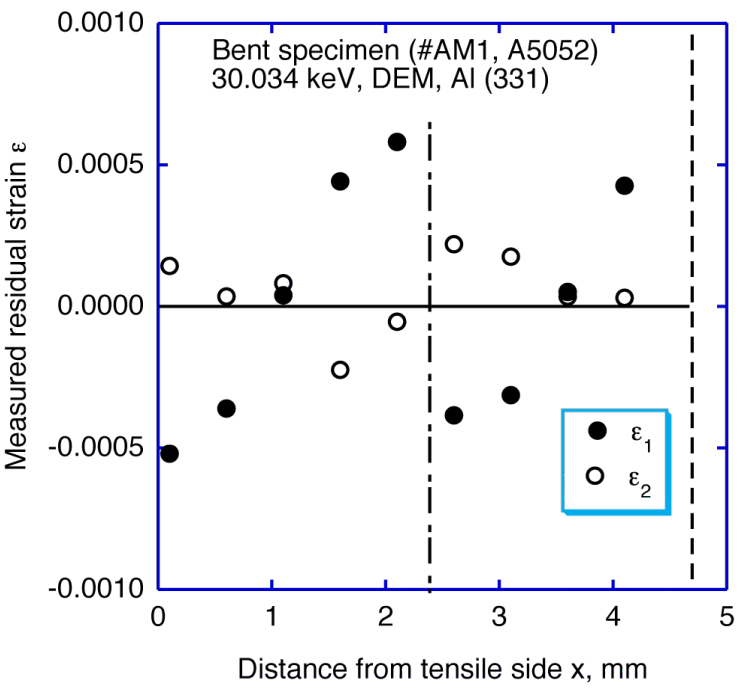

(a) Residual strain

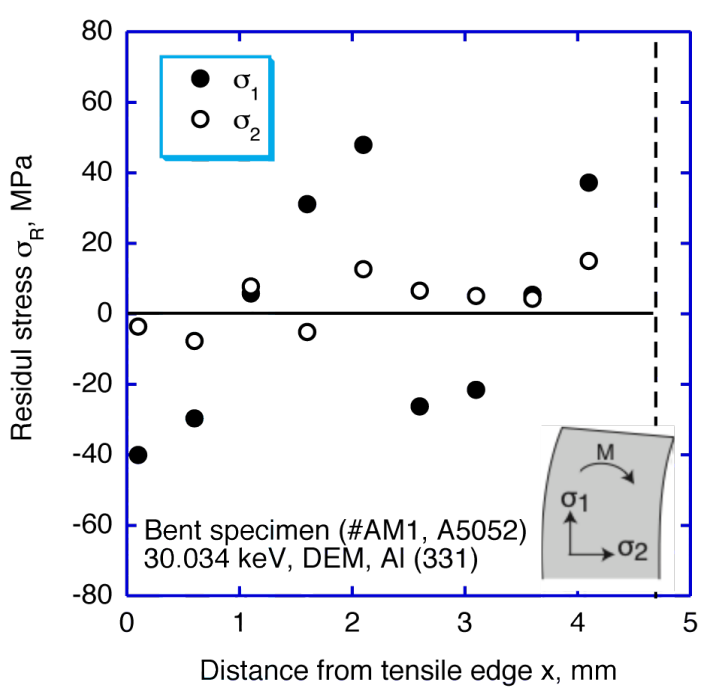

(b) Residual stress

Fig. 4 Residual strain and stress of the plastically bent specimen measured by DEM.

The distributions of the residual strain and stress are shown in Fig. 4 (a) and (b). The strain $\varepsilon_{1}$ and the stress $\sigma_{1}$ are along the longitudinal axis, while the strain $\varepsilon_{2}$ and the stress $\sigma_{2}$ are along the 
width of the bar. The stresses were determined from the strains for the bent specimen under the biaxial stress assumption. The compressive residual strain and stress are introduced in the tensile side of bending, and these results show the typical distribution of the plastically bent plate.

\section{Residual Stress Distributions of the Indented Specimen}

To assess the effectiveness of the DEM, we measured the residual stress distribution of the indented specimen. A rectangular bar was used to strike the side edge of the plate specimen twice, as shown in Fig. 5 (a). The $1^{\text {st }}$ indentation was tilted to the left, and the $2^{\text {nd }}$ indentation was driven vertically. Figure 5 (b) shows the indented specimen, and the distribution of the residual stress around the asymmetric indentation is interesting.

Figure 6 (a) shows the stress maps of the indented specimen measured by the DEM. The measured area was $8-\mathrm{mm}$ long and $4.7-\mathrm{mm}$ wide. The diffraction angle with respect to the horizontal and vertical directions was measured every $0.2 \mathrm{~mm}$. The incident X-ray beam penetrated through a thickness of the specimen, and the diffraction angles with $\chi=0^{\circ}$ and $90^{\circ}$ were measured.

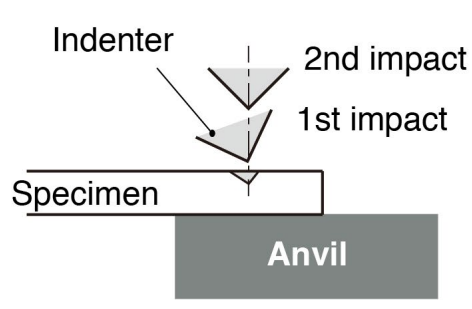

(a) Making of indentation

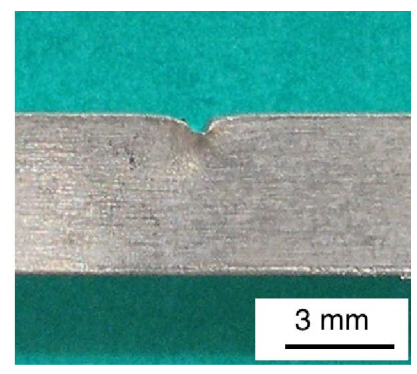

(b) Indented specimen Fig. 5 Indentation of the specimen.

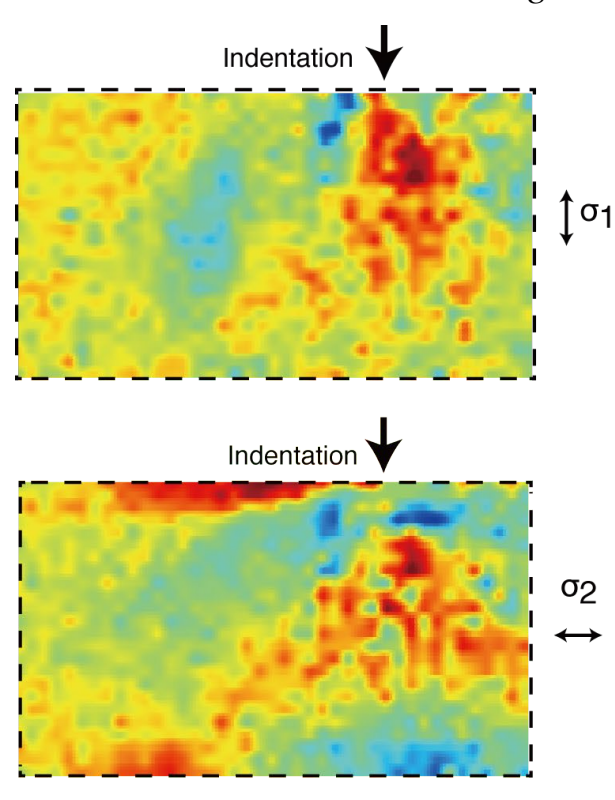

(a) Measured by DEM

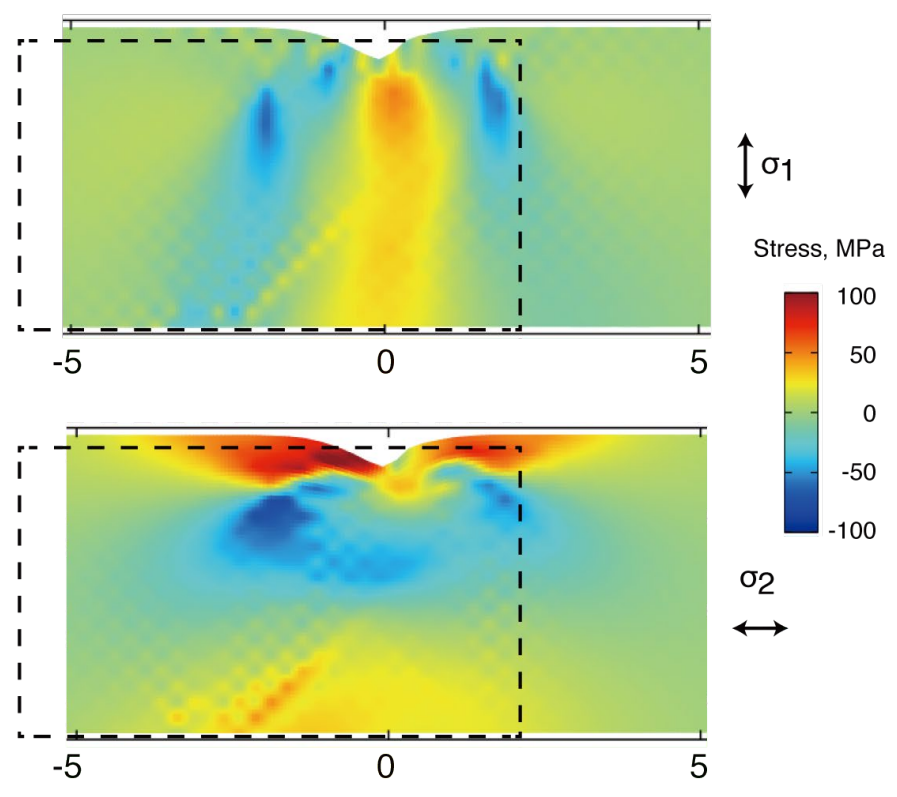

(b) Simulated by FEM

Fig. 6 Distribution of the residual stress in the indented specimen.

A plane stress regime was assumed, and the stress and strain were obtained from the measured diffraction angles. For the residual stress $\sigma_{1}$, a large tension is introduced under the indentation, and a compressive region exists around the indentation, as shown in Fig. 6 (a). For the residual stress $\sigma_{2}$, a large tensile region was generated in the left side of the indentation, and 
compression is introduced under the indentation. A tensile region was introduced near the edge of the anvil.

A simulation of a model with a similar indentation was performed to verify the accuracy of the obtained residual stress distribution. The simulation was performed with the finite element program "Impact" [9], and the results are shown in Fig. 6 (b). The broken line in the figure indicates the area measured by the DEM. When Fig. 6 (a) is compared with Fig. 6 (b), the measured residual stress distribution is similar to the simulated one, indicating that the DEM is useful to measure the stress of coarse grain materials.

\section{Summary}

A DEM using hard synchrotron X-rays was proposed. The residual stresses of a plastically bent specimen and the residual stress distribution within an indented specimen were measured, and the accuracy of the method was verified by comparison with simulated data. The results show that the DEM is useful for the stress measurement of coarse grain materials.

\section{Acknowledgements}

The authors would like to acknowledge the financial support from the Japan Society for the Promotion of Science, JSPS KAKENHI for Scientific Research (C) 17K06046. The synchrotron radiation experiment on the BL22XU beamline at SPring-8 was performed with the approval of the JAEA (No. 2017A-E10), and the experiment at BL22XU was supported by the "Nanotechnology Platform" of the Japanese Ministry of Education, Culture, Sports, Science and Technology.

\section{References}

[1] B.B. He, K.L. Smith, A new method for residual stress measurement using an area detector, in: T. Ericsson, M. Odén, A. Andersson (Eds), Proc. of the 5th international conference on residual stresses (ICRS-5), Linköping, Sweden, 1997, pp. 634-639.

[2] S. Taira, K. Tanaka, Local residual stress near fatigue crack tip, Transactions of Iron and Steel Institute of Japan, 19 (1979) pp. 411-418.

[3] K. Suzuki, Proposal for a direct-method for stress measurement using an X-ray area detector, NDT and E International, 92 (2017) pp. 104-110.

https://doi.org/10.1016/j.ndteint.2017.07.012

[4] K. Suzuki, T. Shobu, A. Shiro and S. Zhang, Internal stress measurement of weld part using diffraction spot trace method, Materials Science Forum, 777 (2014) pp. 155-160. https://doi.org/10.4028/www.scientific.net/MSF.777.155

[5] K. Suzuki, T. Shobu, A. Shiro and H. Toyokawa, Evaluation of internal stresses using rotating-slit and 2D detector, Materials Science Forum, 772 (2014) pp. 15-19. https://doi.org/10.4028/www.scientific.net/MSF.772.15

[6] G.N. Kamm and G.A. Alers, Low-temperature elastic moduli of aluminum, Journal of Applied Physics, 35 (1964) pp. 327-330. https://doi.org/10.1063/1.1713309

[7] E. Kröner, Berechnung der elastischen Konstanten des Vierkristalls aus den Konstanten des Einkristalls, Zeitschrift Physik, 151 (1958) pp. 504-518. https://doi.org/10.1007/BF01337948

[8] Information on http://www.rigaku.co.jp/app/kroner/kroner_c.html

[9] Information on https://sourceforge.net/projects/impact/ 